\title{
Measurement and Modeling of Void Fraction in High Pressure Condensing Flows through Microchannels
}

\author{
Brendon KEINATH ${ }^{1}$, Srinivas GARIMELLA ${ }^{1, *}$ \\ * Corresponding author: Tel.: +1 404-897-7479; Fax:+1 404-894-8496; Email: sgarimella@gatech.edu \\ 1: G. W. W. School of Mechanical Engineering, Georgia Institute of Technology, Atlanta, GA, USA
}

\begin{abstract}
Void fraction measurements are obtained using high speed video for the condensation of R404A in tubes of diameter $0.508,1.00$, and $3.00 \mathrm{~mm}$. Experiments were conducted on refrigerant R404A throughout the entire condensation quality range $(0.05<x<0.95)$ at varying mass fluxes $\left(200 \leq G \leq 800 \mathrm{~kg} \mathrm{~m}^{-2} \mathrm{~s}^{-1}\right)$ and saturation temperatures from 30 to $60^{\circ} \mathrm{C}\left(0.38 \leq p_{\mathrm{r}} \leq 0.77\right)$. These high pressures are representative of actual operation of air-conditioning and refrigeration equipment. The influence of saturation temperature on void fraction is most pronounced in the quality range $0.25<x<0.75$. In addition, it was found that the influence of mass flux on void fraction was negligible for all saturation temperatures and tube diameters investigated. A new drift flux void fraction model is developed to predict void fraction for condensing flows in microchannels and compared with the R404A data and R134a void fraction data from Winkler et al. (2012a). Overall the model is able to predict $92.3 \%$ of the R404A data and $81.6 \%$ of all refrigerant data within $25 \%$.
\end{abstract}

Keywords: Microchannel, Condensation, Void Fraction, Visualization, Refrigerants

\section{Introduction}

For in-tube condensation, the void fraction concept is used to infer the thickness of the liquid film and is a building block for condensation pressure drop and heat transfer models. Thus, there is a need for accurate correlations for void fraction under varying flow conditions, particularly in mini- and microchannels. Most of the existing studies in small diameter tubes have utilized air-water experiments for the development of void fraction models. While these investigations provide a good baseline understanding, extrapolation of results to fluids with drastically different properties must be done cautiously. Furthermore, the influence of flow regimes in defining the vapor-liquid interface is often overlooked.

A comprehensive understanding of the effects of fluid properties and tube diameter on void fraction in condensing refrigerants is required to accurately model the transport of heat, mass and momentum. Void fraction is also important for estimating the charge of refrigerant containing systems. The present study obtains carefully measured void fractions over a wide range of condensation conditions and develops a drift-flux model that accurately predicts measured void fraction data.

\section{Previous Work}

Early void fraction work in two phase flow was largely focused on steam and air-water mixtures. Several different techniques have been used to measure void fraction, including radiation attenuation and neutron radiography, electrical impedance, shut-off valves, and flow visualization with image analysis. A comprehensive review of void fraction literature including experimental techniques and correlations is presented in Winkler et al. (2012b).

Many of the flow visualization studies in the literature have used air-water mixtures as the working fluid. However, the phase properties of air-water and refrigerant vapor-liquid phases are quite different, specifically density ratios and surface tension, which makes extrapolation from air-water to refrigerant flows difficult. One of the major challenges limiting condensation void fraction experiments is the high pressures associated with saturation temperatures representative of typical heat rejection conditions. These challenges were 
overcome in the study by Coleman and Garimella (2003), who obtained video recordings of condensing $\mathrm{R} 134 \mathrm{a}$ in round, square and rectangular channels with hydraulic diameters ranging from $1-4.91 \mathrm{~mm}$. Representative saturation temperatures were achieved by flowing compressed air through the annulus of the test section, which minimized the differential pressure subjected on the glass tube and also achieved controlled condensation of the fluid in the test section. A similar technique is used here.

Jassim et al. (2008) developed a void fraction model based on their probabilistic flow regime map for various refrigerants $(R 11, R 12$, $\mathrm{R} 134 \mathrm{a}, \mathrm{R} 22, \mathrm{R} 410 \mathrm{~A}$ ) in circular tubes ranging from 4.26 to $9.58 \mathrm{~mm}$ in diameter. The data used by them include condensing, adiabatic and evaporating two-phase flow. The model is based on three specific models for annular, stratified, and intermittent flow, and uses weighting functions to account for the probability that each flow regime exists for the specific flow parameters. This approach yields a continuous function but is primarily based on statistical fits, and may not model the phenomena accurately, because it is unlikely that intermittent, stratified, and annular flow can exist for the same flow conditions within the same tube.

More recently, Winkler et al. (2012a) developed an image analysis program to obtain condensation void fraction in the wavy and intermittent flow regimes from the video recordings of Coleman and Garimella (2003). It was found that void fraction in the wavy flow regime depended strongly on quality, but showed little sensitivity to hydraulic diameter and mass flux. However, these trends were not as clearly observed in the intermittent or intermittent-wavy overlap regime. From their data, they developed a flow regime specific drift-flux model. It was difficult to define the distribution parameter and vapor drift flux with relavant two-phase flow parameters; therefore, a best fit using empirical constants was chosen.

\section{Experimental Approach}

In the present study, two separate experimental facilities (one for large $(3 \mathrm{~mm}$ ) and another for small (1, $0.5 \mathrm{~mm})$ channels) were used to obtain simultaneous high-speed flow visualization and pressure drop measurements for a wide range of tube diameters, mass fluxes and saturation temperatures with refrigerant R404A. More complete descriptions of the large tube (Coleman and Garimella, 2003) and the small tube (Agarwal and Garimella, 2010) facilities are provided elsewhere.

The test section in both facilities was a counter-flow tube-in-tube condenser. A photograph and schematic of a test section are shown in Figures 1(a) and 1(b), respectively. The refrigerant flowed through the inner tube and compressed cold air flowed through the annulus. This allowed for condensation during the visualization while maintaining a small pressure difference across the glass tube, which enabled testing at high saturation pressures. The flow rate of the air was controlled to achieve a change in quality, $\Delta x$, of about 0.05 across the test section. High-speed video was recorded using a Photron FASTCAM Ultima 1024. Images were recorded using a microscopic lens. For the $\mathrm{D}=$ $3 \mathrm{~mm}$ tube, a Nikon micro-Nikkor $105 \mathrm{~mm}$ lens was used, while a Navitar $12 \mathrm{X}$ lens capable of 0.58 to $7 \mathrm{X}$ magnification was used for the smaller channels. The resolution for the field of views for each tube was $512 \times 1096$. The images recorded in this study were taken at $1,000 \mathrm{fps}$ for a duration of 1 second, resulting in 1,000 frames per data point for analysis.

\section{Analysis}

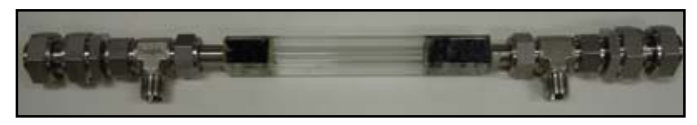

(a)

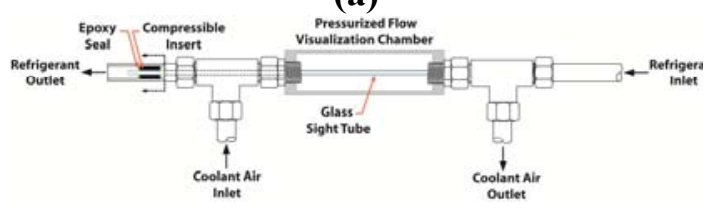

(b)

Fig. 1. A (a) photograph and (b) schematic of the 3 $\mathrm{mm}$ visualization test section 


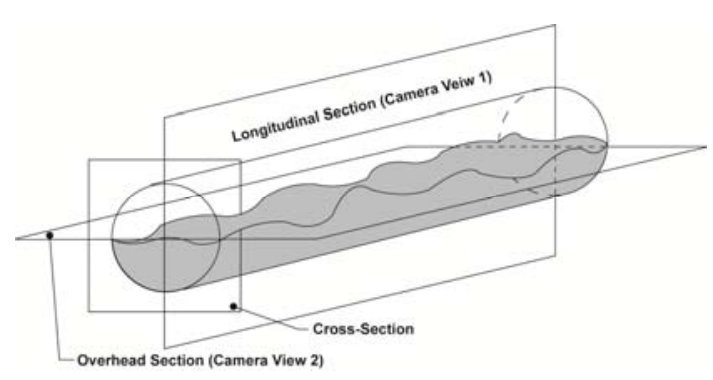

Fig. 2. Observed planes: longitudinal, overhead, and cross-section

In the present study, images of the longitudinal section of the tube defined in Figure 2 are taken to provide quantitative void fraction data. To obtain three-dimensional information from the two-dimensional images obtained in the video recordings, several geometric assumptions were made. A second image perpendicular to the longitudinal section (i.e., the overhead section) was also taken to validate these assumptions.

An image analysis tool was developed inhouse using edge-detection capabilities available in the MATLAB (The MathWorks Inc., 2004c) platform. The tool was originally developed for analyzing falling-films and droplets flowing around tube banks. In the present study, this original tool was modified and enhanced to obtain liquid-vapor phase distributions and void fractions. A detailed description of the development of this imageanalysis tool is provided in Killion and Garimella (2004).

The program uses a graphical user interface (GUI) to allow for the video recordings to be analyzed in a frame-by-frame manner. The program automatically detects the edge of the vapor-liquid interface within a user-defined region of interest (ROI), sets points along the path of the detected edges, and fits a smoothing spline through these points. The program uses the Canny edge-detection algorithm from the MATLAB Image Processing Toolbox (The MathWorks Inc., 2004a) combined with the cubic smoothing spline function SPAPS from the MATLAB Spline Toolbox (The MathWorks Inc., 2004b).

\subsection{Identification of Flow Characteristics}

The vapor bubble parameters and void

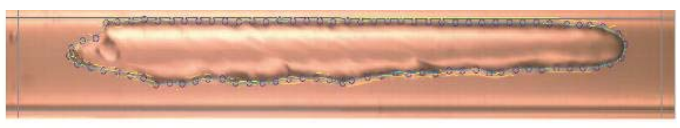

(A)



(B)

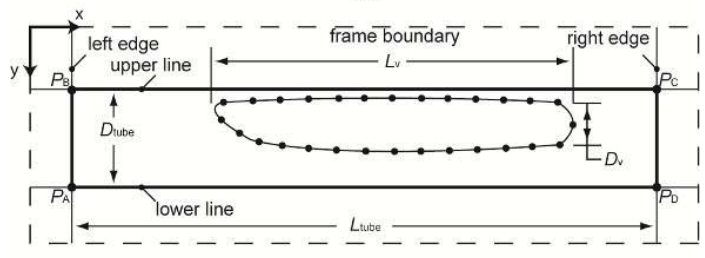

(C)

Fig. 3. Schematic of the geometric features in a video frame

fraction are calculated based on the spline fit to the vapor-liquid interface and the boundaries of the tube, which are user inputs. An example frame analysis and schematic are shown in Figures 3a-c, with the pixel coordinate system defined in the upper left corner. This coordinate system is used to define the outline of the tube diameter, $D$, using the upper and lower lines, and also the tube length, $L_{\text {tube, }}$ using the left and right boundary lines. The boundary lines are fully adjustable and are set by the user. The number of pixels per millimeter, $x_{\mathrm{cal}}$, is calibrated to the known tube diameter. This user input procedure is carried out in the first frame only and then automatically updated throughout the analysis. For a tube diameter of $D=3 \mathrm{~mm}$, the length of the tube captured in the image was typically $L_{\text {tube }}=22 \mathrm{~mm}$, and a calibration value of $x_{\text {cal }}=$ 45 pixels/mm was used.

\subsection{Calculation of Void Fraction}

The data were classified into one of four major flow regimes: annular, wavy, intermittent, and dispersed. A detailed description of each flow regime and the corresponding flow patterns is available in Coleman and Garimella (2003). The twodimensional image is then used to calculate the void fraction based on the liquid-vapor interface geometry for the different flow regimes. Two different calculation methodologies are used, described below. The vapor inventory in each frame is also 
captured in two dimensions. If more than one continuous vapor bubble is present in a frame, the vapor volume in each vapor bubble is calculated separately and then summed to determine the volumetric void fraction for each frame.

The resulting void fraction is a function of the geometric assumptions made about the cross-sectional area. These assumptions were validated using alternate viewing angles, obtained for representative cases at a range of quality, mass flux and tube diameters. For example, the Overhead Section view (Figure 2) provided a view of the condensing fluid from the top, which enabled a realistic construction of the three-dimensional shape of the vapor and liquid features. The cross sectional geometry is either assumed to be a circle, or a partial circle. Analysis of the different vapor phase geometries is described below, followed by the methodology for calculating local, volumetric, and time-averaged void fractions.

\section{Cylindrical Vapor Geometry}

The cylindrical vapor geometry assumption assumes that the vapor inventory flowing through the tube has a circular cross-section. Therefore, the average vapor diameter, $D_{\mathrm{v}, \mathrm{i}}$, for each segment is used to calculate the crosssectional area, $A_{\mathrm{cs}, \mathrm{i}}$.

$$
A_{\mathrm{cs}, \mathrm{i}}=\frac{\pi}{4} D_{\mathrm{v}, \mathrm{i}}^{2}
$$

The cylindrical geometry assumption is typically valid for small tube diameters where gravitational influences are relatively small. The vapor bubble is modeled as a finite number of cylinders stacked next to each other. This assumption was found to be valid for intermittent and annular flows.

\section{Partial Cylinder Geometry}

In the wavy flow regime, the effects of an increased liquid inventory at the bottom of the tube must be considered to predict the volumetric void fraction from the twodimensional image. This deviation from a symmetrical, circular cross section is accounted for by assuming a partial-cylinder geometry (Figure 4). In this case, the thickness of the



Fig. 4. Cross-sectional areas of partial cylinder and cylindrical vapor phase cases

film located at the top of the tube is calculated using the upper line, $y_{\mathrm{UL}}$, that defines the tube diameter (Figure 4) and the average upper edge, $y_{\text {avg,upper,i, for each segment: }}$

$$
t_{\text {film, i }}=y_{\mathrm{UL}}-y_{\text {avg, upper,i }}
$$

In the case of completely stratified flow, the film thickness at the top would be zero. The radius, $R$, of the partial-cylinder shape is defined as:

$$
R=\frac{D_{\text {tube }}}{2}-t_{\text {film }}
$$

This radius of the vapor core is shown in Figure 4. Defining the radius in this manner may slightly over predict the actual vapor inventory as the region represented by the shaded partial circle in Figure 4 is probably not centered on the tube axis, leading to a variation of the film thickness around the circumference. However, this approach does account well for the more significant issue of the thicker pool of liquid in the stratified region at the bottom. There are three different cases for the calculation of the cross-sectional area of the vapor inventory, determined by the vertical distance of the bottom liquid-vapor interface, $H$, from the tube centerline.

\subsection{Void Fraction Measurement}

The volume of the vapor inventory for one segment, $V_{\mathrm{v}, \mathrm{i}}$, can be calculated once the crosssectional area for the segment is computed as follows:

$$
\langle\alpha\rangle=\frac{V_{\mathrm{v}, \mathrm{i}}}{V_{\text {tube, } \mathrm{i}}}=\frac{A_{\mathrm{cs}, \mathrm{i}} t_{\text {segment }}}{(\pi / 4) D_{\text {tube }}^{2} t_{\text {segment }}}=\frac{A_{\mathrm{cs}, \mathrm{i}}}{(\pi / 4) D_{\text {tube }}^{2}}
$$

The local void fraction is a function of axial position. The volumetric void fraction, $\langle\langle\alpha\rangle\rangle$ 


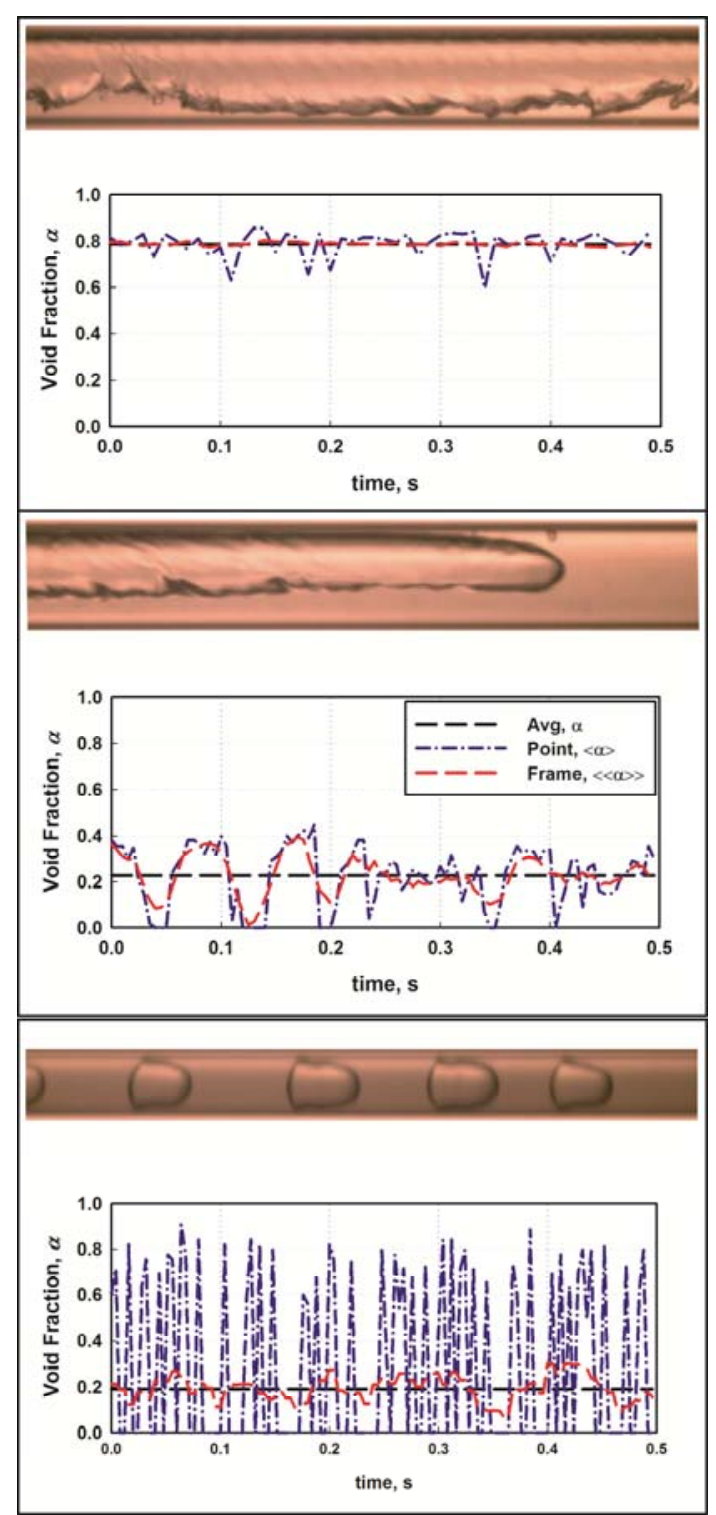

Fig. 5. Void fraction as a function of time for each frame: center point (blue), volume (red)

(void fraction for 1 frame,) is measured by summing all of the local vapor volumes along the tube and dividing by the tube volume.

$$
\langle\langle\alpha\rangle\rangle=\frac{V_{\mathrm{v}}}{V_{\text {tube }}}=\frac{\sum_{i=1} V_{\mathrm{v}, \mathrm{i}}}{(\pi / 4) D_{\text {tube }}^{2} L_{\text {tube }}}
$$

The void fraction is calculated on a frame-byframe basis but the time and volume average is of interest to determine the bulk average void fraction.

$$
\alpha=\langle\langle\bar{\alpha}\rangle\rangle=\frac{\sum_{j=1}^{N}\langle\langle\alpha\rangle\rangle_{j}}{N}
$$

The local, $\langle\alpha\rangle$ (blue,) volumetric, $\langle\langle\alpha\rangle\rangle$ (red,) and volume and time averaged, $\alpha$ (black,) void fractions for three representative analyses are shown in Figure 5. The top and middle plot demonstrate the difference in dynamic signal as a function of flow regime in the same tube diameter $(D=3 \mathrm{~mm}$.) The local and volumetric values for the wavy flow example do not deviate significantly from the average value. It is difficult to differentiate between the volume and volume-time average values. The intermittent signal varies significantly from the average value due to the nature of the flow regime. The vapor bubbles are intermittently separated by liquid slugs, which result in a local measured void fraction of 0 . The slug frequency can be inferred from this plot. The void fraction measures 0 at six different discrete times over the $0.5 \mathrm{~s}$ period resulting in a measured slug frequency of $\omega=12 \mathrm{~s}^{-1}$. The bottom plot demonstrates the influence of tube diameter $(D=1 \mathrm{~mm})$ on measured void fraction for the same flow regime. The bulk average for the middle and bottom plot are similar, $\alpha=$ 0.21 , but the dynamic signals are quite different. The decreased tube diameter results in a substantial increase in vapor bubble velocity and frequency. The experimental methodology was validated by comparing the still images with known reference volumes.

\subsection{Total Uncertainty}

The total uncertainty in the void fraction was estimated using a root mean square of all of the major uncertainties in the measurements and analyses. The total uncertainty accounts for the uncertainty of the average test section quality from experiments, $U_{x, \exp }$, deviations introduced by the selection of the number and frequency of frames analyzed, $U_{\text {sens, }}$ and the repeatability of the process, $U_{\text {rep}}$.

$$
U_{\text {tot }}=\sqrt{U_{x, \exp }^{2}+U_{\text {sens }}^{2}+U_{\text {rep }}^{2}}
$$

The uncertainty related to the sensitivity analysis is taken as the percentage deviation for 
the given flow regime and tube diameter, while the repeatability uncertainty is taken as 3 times the measured standard deviation. The average uncertainty in void fraction over the entire range of qualities for which the tests were conducted was found to be $10.3 \%$, with the minimum uncertainty of $2 \%$ occurring at $\alpha=$ $0.86(x=0.57$,$) and the maximum uncertainty$ of $40.5 \%$ occurring at $\alpha=0.22(x=0.10$. $)$

\section{Results and Discussion}

Condensation void fraction results were obtained for tube diameters of $0.508,1.00$, and $3.00 \mathrm{~mm}$ at mass fluxes and saturation temperatures ranging from 200 to $800 \mathrm{~kg} \mathrm{~m}^{-2} \mathrm{~s}^{-}$ ${ }^{1}$ and 30 to $60^{\circ} \mathrm{C}$, respectively, for a total of 142 void fraction data points. Figure 6(a) shows void fraction as a function of quality for varying saturation temperatures for $D=3 \mathrm{~mm}$ and $G=$ $200 \mathrm{~kg} \mathrm{~m}^{-2} \mathrm{~s}^{-1}$. For all saturation temperatures, the void fraction approaches unity as the flow becomes completely vapor and approaches 0 as the flow becomes completely liquid. At both extremes, the measured results approach the homogeneous flow model. The influence of saturation temperature on void fraction is most pronounced in the quality range $0.25<x<0.75$. As the saturation temperature increases, the liquid-vapor density ratio decreases, decreasing the vapor volume at a given quality. The influence of mass flux on void fraction is shown in Figure 6(b) for a representative case with $D$ $=3 \mathrm{~mm}$ and $T_{\text {sat }}=35^{\circ} \mathrm{C}$. It was found that the influence of mass flux was negligible for all saturation temperatures and tube diameters investigated.

Figure 6(c) shows a plot of void fraction versus quality at $T_{\mathrm{sat}}=40^{\circ} \mathrm{C}$ for $D=0.508,1.00$, and $3.00 \mathrm{~mm}$. It can be seen that throughout the condensation process, the measured void fraction is over predicted by the homogeneous flow void fraction expression, and also that the void fraction is insensitive to hydraulic diameter. However, even though the vaporto-liquid volume ratio appears unaffected by tube diameter and flow regime, as the hydraulic diameter changes, surface tension affects the distribution of the vapor-liquid inventory. Figure 7(a) shows the effects of tube diameter

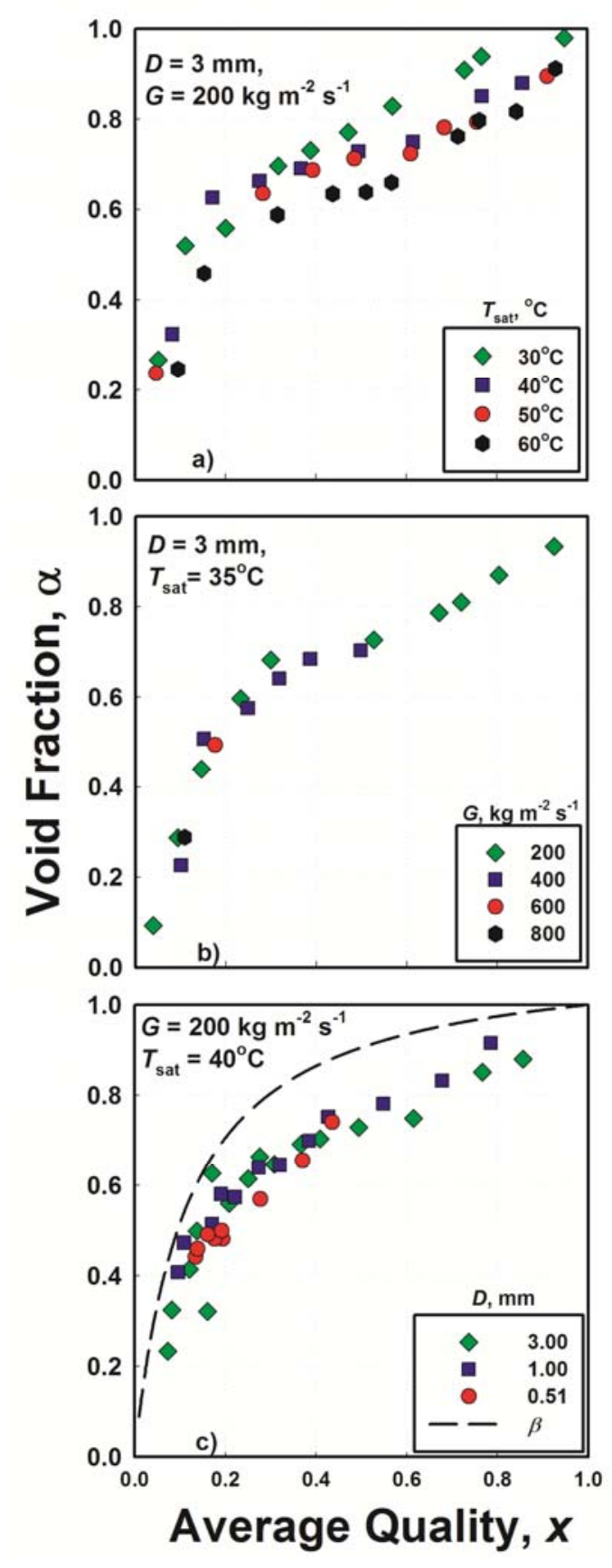

Fig. 6. Experimental void fraction versus quality for different saturation temperatures (a), mass fluxes (b), and diameters (c)

on vapor-liquid distribution under similar conditions $\left(T_{\text {sat }}=30^{\circ} \mathrm{C}, G=200 \mathrm{~kg} \mathrm{~m}^{-2} \mathrm{~s}^{-1}\right)$. In both cases, the interface has been tracked to allow for quantitative evaluation, with a resulting average void fraction of approximately 0.52 for both tubes. However, for $D=3.00 \mathrm{~mm}$, there is stratified liquid 
(a)

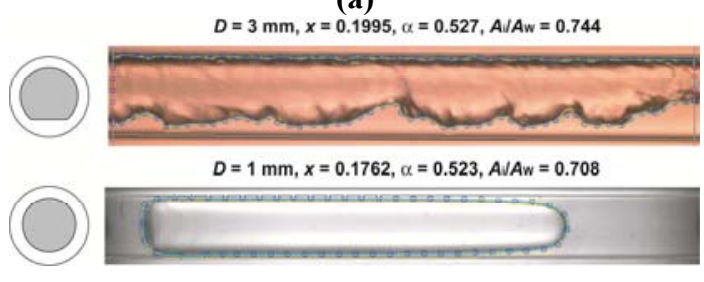

(b)

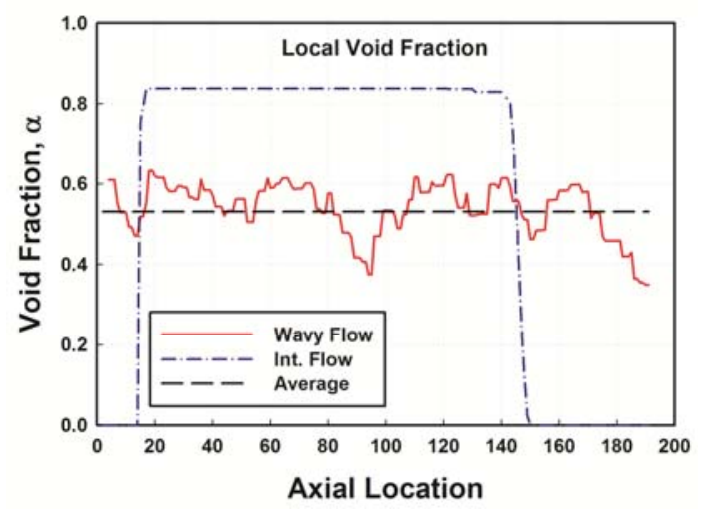

Fig. 7. Comparison of vapor-liquid distribution for different $D$ on a (a) frame and (b) local basis for $T_{\text {sat }}$ $=30^{\circ} \mathrm{C}, G=200 \mathrm{~kg} \mathrm{~m}^{-2} \mathrm{~s}^{-1}$

inventory corresponding to the wavy flow regime for an idealization using a partial circle cross-section. The film thickness in this case varies azimuthally. The interfacial area, $A_{\mathrm{i}}$, to wetted tube area, $A_{\mathrm{w}}$, ratio is 0.744 . For $D=$ $1.00 \mathrm{~mm}$, the flow is in the intermittent regime and the interfacial area is limited to the vapor bubble surface. The ratio of $A_{\mathrm{i}}$ to $A_{\mathrm{w}}$ for this distribution is 0.708 . The different interfacial area ratios in these cases, in conjunction with the respective phase velocities, determine pressure drop and heat transfer. The local void fraction for each example frame is shown in Figure 7(b). Despite the bulk averages being approximately the same, it is clear that the local void fraction distribution differs substantially. These different distributions would lead to different vapor-liquid transport mechanisms and also different velocity profiles within each phase.

The void fraction data from the present study are compared with three models from the literature that are frequently used in microchannel applications. The comparisons are categorized by flow regime and a summary of the absolute average deviation (AAD) is presented in Table 1. While these three correlations demonstrate a reasonable overall $\mathrm{AAD}$, it should be noted that they predict all annular data well, but fail to accurately capture the trends in void fraction in the intermittent and intermittent-wavy flow regimes. Furthermore, the Baroczy (1965) correlation, which predicts the data the best, provides nonphysical behavior at high reduced pressure due to a non-linear dependence on quality ratio (i.e. as the critical point is approached, the void fraction value predicted will not approach 1). Thus, a new, physically consistent model is introduced in the following section.

\section{Void Fraction Model}

The vapor drift flux velocity was used to derive the void fraction model. The derivation for the vapor drift flux velocity was conducted based on an approach similar to that presented by Ishii et al. (1976). Assuming equal pressure gradient in both phases, the following result is obtained:

$$
\begin{aligned}
& 0=\frac{\tau_{\text {wall }} P_{\text {wall }}}{A(1-\alpha)}-\frac{\tau_{\mathrm{i}} P_{\mathrm{i}}}{A}\left[\frac{1}{\alpha}+\frac{1}{1-\alpha}\right] \\
& =\frac{\tau_{\text {wall }} P_{\text {wall }}}{A(1-\alpha)}-\frac{\tau_{\mathrm{i}} P_{\mathrm{i}}}{A \alpha(1-\alpha)}
\end{aligned}
$$

Table 1. Average absolute difference of void fraction models in literature with data by observed flow regime

\begin{tabular}{|l|l|l|l|l|l|l|}
\hline Model & Int. & $\begin{array}{l}\text { Int.- } \\
\text { Wavy }\end{array}$ & Wavy & Int.-Ann. & Annular & Overall \\
\hline \# of Data Points & 38 & 4 & 49 & 25 & 26 & $\mathbf{1 4 2}$ \\
\hline Homogeneous & $47.8 \%$ & $57.1 \%$ & $24.4 \%$ & $24.6 \%$ & $16.8 \%$ & $\mathbf{3 0 . 2 \%}$ \\
\hline Armand (1946) & $26.9 \%$ & $30.8 \%$ & $7.9 \%$ & $6.3 \%$ & $5.5 \%$ & $\mathbf{1 2 . 9 \%}$ \\
\hline Baroczy (1965) & $21.9 \%$ & $20.9 \%$ & $8.0 \%$ & $5.7 \%$ & $5.3 \%$ & $\mathbf{1 1 . 2 \%}$ \\
\hline El Hajal et al. $(2003)$ & $36.6 \%$ & $43.3 \%$ & $18.4 \%$ & $17.8 \%$ & $13.6 \%$ & $\mathbf{2 3 . 0 \%}$ \\
\hline
\end{tabular}


By rearranging and balancing the interfacial and wall shear forces, the resulting equation becomes:

$$
\frac{\tau_{\mathrm{i}} P_{\mathrm{i}}}{\alpha}=\tau_{\text {wall }} P_{\text {wall }}
$$

To determine a closed form solution, the shear stresses in the liquid film and at the interface should be defined. The film shear stress is defined as:

$$
\tau_{\text {wall }}=\frac{f_{\mathrm{L}} \rho_{\mathrm{L}} U_{\mathrm{L}}\left|U_{\mathrm{L}}\right|}{2}
$$

The interfacial shear stress is defined as:

$$
\tau_{\mathrm{i}}=\frac{f_{\mathrm{i}} \rho_{\mathrm{v}} U_{r}^{2}}{2}
$$

where $U_{\mathrm{r}}$ is the relative velocity between the two phases and can be related to the vapor drift flux velocity using the following relation:

$$
\bar{V}_{\mathrm{vj}}=U_{\mathrm{v}}-j=(1-\alpha) U_{\mathrm{r}}
$$

Substituting Equations (10) and (11) into (9) , the force balance can be written in terms of velocities and friction factors:

$$
\left(\frac{f_{\mathrm{i}} \rho_{\mathrm{v}} U_{\mathrm{r}}^{2}}{2}\right) \frac{P_{\mathrm{i}}}{\alpha}=\left(\frac{f_{\mathrm{L}} \rho_{\mathrm{L}} U_{\mathrm{L}}\left|U_{\mathrm{L}}\right|}{2}\right) P_{\text {wall }}
$$

Rearranging Equation (13) and solving for the relative velocity, $U_{\mathrm{r}}$.

$$
U_{\mathrm{r}}^{2}=\left(\frac{f_{\mathrm{L}}}{f_{\mathrm{i}}}\right)\left(\frac{\rho_{\mathrm{L}}}{\rho_{\mathrm{v}}}\right)\left(\frac{P_{\mathrm{wall}}}{P_{\mathrm{i}}}\right) U_{\mathrm{L}}\left|U_{\mathrm{L}}\right| \alpha
$$

The liquid film velocity can be approximated using the relationship:

$$
U_{\mathrm{L}}=j_{\mathrm{L}} /(1-\alpha)
$$

The vapor drift flux velocity can then be approximated by:

$$
\bar{V}_{\mathrm{vj}}^{2}=\left(\frac{f_{\mathrm{L}}}{f_{\mathrm{i}}}\right)\left(\frac{\rho_{\mathrm{L}}}{\rho_{\mathrm{v}}}\right)\left(\frac{P_{\mathrm{wall}}}{P_{\mathrm{i}}}\right) \alpha j_{1}^{2}
$$

The interfacial friction has been predicted by previous researchers in two-phase flow. Wallis (1969) developed one of the original equations for interfacial friction and more recently, Garimella et al. (2005) developed a formulation specific to condensing flows in small diameter channels:

$$
\frac{f_{\mathrm{i}}}{f_{\mathrm{L}}}=A \cdot X^{a} \operatorname{Re}_{\mathrm{L}}^{b}\left(\frac{C a_{\mathrm{L}}}{1-\alpha}\right)^{c}
$$

The interfacial friction factor ratio was defined by Garimella et al. (2005) as a function of the Martinelli parameter, $X$, the liquid Reynolds number, ReL, and the liquid Capillary number, CaL. The resulting vapor drift flux velocity equation can then be interpreted in terms of the parameters:

$$
\bar{V}_{\mathrm{vj}}=f\left(X, \operatorname{Re}_{\mathrm{L}}, C a_{\mathrm{L}}, \sqrt{\frac{\rho_{\mathrm{L}}}{\rho_{\mathrm{v}}}}, \sqrt{\frac{P_{\mathrm{wall}}}{P_{\mathrm{i}}}}, \sqrt{\alpha}, j\right)
$$

It was observed that the bulk measured void fraction was not heavily dependent on diameter, mass flux, or flow regime. Therefore, the Reynolds number, and perimeter ratio are not considered to be particularly significant, and are excluded from the formulation. It was also deemed that any minor gains in accuracy by retaining void fraction as a dependent variable would be offset by the requirement of an iterative solution; therefore, the void fraction was also excluded from the formulation. It is hypothesized that the vapor drift flux velocity should approach the homogeneous void fraction as the critical pressure is approached; therefore, the density ratio term is included as shown in the brackets in Equation (19). Thus, as the critical pressure is approached, the density ratio tends to unity and the drift flux will approach zero. The simplified formulation for the vapor drift velocity equation becomes:

$$
\bar{V}_{\mathrm{vj}}=A \cdot X^{b} \cdot C a_{\mathrm{L}}^{c} \cdot\left(\sqrt{\frac{\rho_{\mathrm{L}}}{\rho_{\mathrm{v}}}}-1\right)^{d} j
$$

A regression analysis was conducted only on data in the annular flow regime $(23 \%$ of data) because the physical considerations are based on this flow mechanism, and the results were extrapolated to data from the other regimes. The resulting equation for the drift flux velocity is: 


$$
\begin{aligned}
\bar{V}_{\mathrm{vj}}= & 0.336 \cdot X^{0.25} \cdot C a_{\mathrm{L}}{ }^{0.154} \\
& \times\left(\sqrt{\frac{\rho_{\mathrm{L}}}{\rho_{\mathrm{v}}}}-1\right)^{0.81} j
\end{aligned}
$$

The vapor drift flux velocity can be related to void fraction as follows:

$$
\alpha=\frac{\beta}{C_{\mathrm{o}}+\bar{V}_{\mathrm{vj}} / j}
$$

In the above equation, the drift flux distribution function $C_{\mathrm{o}}$ is set to 1 , which was found to yield the best agreement over all flow regimes for the data from the present study. The void fraction predictions from this model are shown in Figure 8 . The model predicts $92.3 \%$ of the data within $\pm 25 \%$ with an AAD and $\mathrm{AD}$ of 10.3 and $0.2 \%$ respectively. $\mathrm{A}$ summary of the average deviations is displayed in Table 2. The model predicts all data in the Annular, Disperse Wave, and Intermittent/Annular regime very well. The void fraction model is also compared with previous void fraction data obtained by Winkler et al. (2012a), with the results are shown in Figure 9 . The model predicts $70 \%$ of the R134a data within $25 \%$ with an AAD and AD of 15.6 and $-4.0 \%$ respectively. The model consistently under-predicts these data, which may be a result of the Winkler et al. (2012a) measured void fraction not accounting for the liquid in the upper film.

\section{Conclusions}

A comprehensive investigation of void fractions for condensing high pressure



Fig. 8. Overall void fraction prediction for R404a data

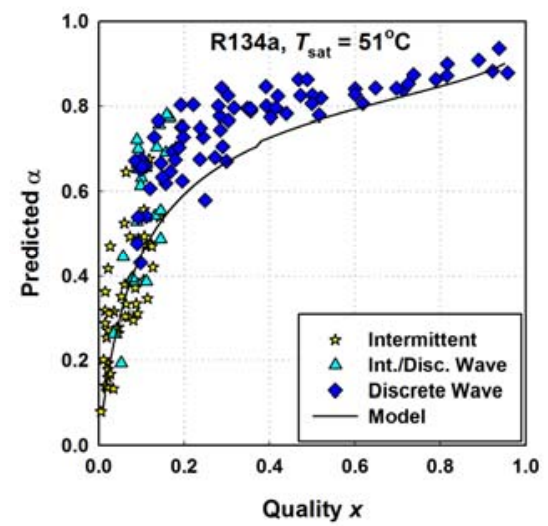

Fig. 9. Void fraction prediction for R134a data from Winkler et al. (2012a)

refrigerant through microchannels was conducted. Experiments were conducted on refrigerant R404A throughout the entire condensation range $(0.05<x<0.95)$ at varying mass fluxes $\left(200 \leq G \leq 800 \mathrm{~kg} \mathrm{~m}^{-2} \mathrm{~s}^{-1}\right)$ and saturation temperatures from 30 to $60^{\circ} \mathrm{C}$. An image analysis program was developed to measure void fraction from the high speed

Table 2. Void fraction model predictions

\begin{tabular}{|c|c|c|c|c|}
\hline Regime & $\begin{array}{c}\text { Data } \\
\text { Points }\end{array}$ & AAD & AD & $\begin{array}{c}\text { Data < 25\% } \\
\text { Deviation }\end{array}$ \\
\hline Intermittent & 7 & 31.4 & 16.0 & 57.1 \\
\hline Int./Annular & 19 & 8.3 & -5.8 & 91.4 \\
\hline Int./Disc. Wave & 17 & 16.3 & -5.8 & 88.2 \\
\hline Disc. Wave & 45 & 11.4 & 4.1 & 88.9 \\
\hline Disp. Wave & 21 & 5.1 & 0.4 & 100.0 \\
\hline Annular & 33 & 5.6 & -2.2 & 100.0 \\
\hline Total & 142 & 10.3 & 0.2 & 92.3 \\
\hline
\end{tabular}


video data. The average uncertainty in measured void fraction was found to be $10.3 \%$. The void fraction data agreed well with the Baroczy (1965) correlation, but due to the physical inconsistencies associated with the model formulation, a new void fraction correlation was developed. The model developed here agreed well with the data (AAD $=10.3 \%, \mathrm{AD}=0.2 \%$ ), predicting $92 \%$ of data within $25 \%$. The model also predicted void fraction data obtained for condensing R134a well (Winkler et al., 2012a), with AAD $=15 \%$, $\mathrm{AD}=4 \%$. Furthermore, the model followed the observed trends and converges to the homogeneous model as the reduced pressure approaches unity. The void fraction model developed in this study can be used to predict interfacial areas and film thickness that are used as the foundation for mechanistic heat and momentum transfer models in condensing flows through microchannels.

\section{References}

Agarwal, A., Garimella, S., 2010. Representative Results for Condensation Measurements at Hydraulic Diameters similar to 100 Microns. Journal of Heat Transfer 132, 112.

Baroczy, C.J., 1965. Correlation of liquid fraction in twophase flow with application to liquid metals. Chemical Engineering Progress Symposium Series 61, 179-191.

Coleman, J.W., Garimella, S., 2003. Two-phase flow regimes in round, square and rectangular tubes during condensation of refrigerant R134a. International Journal of Refrigeration 26, 117-128.

Garimella, S., Agarwal, A., Killion, J.D., 2005. Condensation pressure drop in circular microchannels. Heat Transfer Engineering 26, 28-35.

Ishii, M., Chawla, T.C., Zuber, N., 1976. Constitutive Equation for Vapor Drift Velocity in Two-Phase Annular Flow. AIChE Journal 22, 283.

Jassim, E.W., Newell, T.A., Chato, J.C., 2008. Prediction of refrigerant void fraction in horizontal tubes using probabilistic flow regime maps. Experimental Thermal and Fluid Science 32, 1141-1155.

Killion, J.D., Garimella, S., 2004. Pendant droplet motion for absorption on horizontal tube banks. International Journal of Heat and Mass Transfer 47, 4403-4414.

The MathWorks Inc., 2004a. Matlab Image Processing Toolbox, Ver. 4.2. Matlab Version 7.0.0.19920 Release 14, Natick, MA.

The MathWorks Inc., 2004b. Matlab Spline Toolbox, Ver. 3.2. Matlab Version 7.0.0.19920 Release 14, Natick, MA.
The MathWorks Inc., 2004c. Matlab Version 7.0.0.19920 Release 14. http://www.mathworks.com, Natick, MA.

Wallis, G.B., 1969. One-Dimensional Two-Phase Flow, New York, McGraw-Hill.

Winkler, J., Killion, J., Garimella, S., 2012a. Void fractions for condensing refrigerant flow in small channels. Part II: Void fraction measurement and modeling. International Journal of Refrigeration 35, 246262.

Winkler, J., Killion, J., Garimella, S., Fronk, B.M., 2012b. Void fractions for condensing refrigerant flow in small channels: Part I literature review. International Journal of Refrigeration 35, 219-245. 